\title{
On a Certain Subclass for Multivalent Analytic Functions with a Fixed Point Involving Linear Operator
}

\author{
Asraa Abdul Jaleel Husien ${ }^{1}$ and Qassim Ali Shakir ${ }^{2}$ \\ ${ }^{1}$ Technical Institute, Diwaniya, Al-Furat Al-Awsat Technical University, Iraq \\ e-mail: asraalsade2@gmail.com \\ ${ }^{2}$ College of Computer Science and Information Technology, University of Al-Qadisiyah, Iraq \\ e-mail: qassim4000@gmail.com
}

\begin{abstract}
In the present paper, we study a subclass for multivalent analytic functions with a fixed point $w$ defined in the unit disk $U$ involving linear operator. Also, we obtain coefficient estimates, extreme points, integral representation and radii of starlikeness and convexity.
\end{abstract}

\section{Introduction}

Denote by $\mathcal{A}(p, w)$ the class of functions $f$ of the form:

$$
f(z)=(z-w)^{p}+\sum_{n=1}^{\infty} a_{n+p}(z-w)^{n+p} \quad(p \in \mathbb{N}),
$$

which are analytic in the unit disk $U=\{z \in \mathbb{C}:|z|<1\}$ and $w$ is a fixed point in $U$.

Let $S(p, w)$ denote subclass of $\mathcal{A}(p, w)$ containing of functions of the form:

$$
f(z)=(z-w)^{p}-\sum_{n=1}^{\infty} a_{n+p}(z-w)^{n+p} \quad\left(a_{n+p} \geq 0, p \in \mathbb{N}\right)
$$

Received: August 4, 2019; Accepted: September 12, 2019

2010 Mathematics Subject Classification: 30C45, 30C50.

Keywords and phrases: multivalent function, extreme points, integral representation, linear operator.

Copyright (C) 2019 Asraa Abdul Jaleel Husien and Qassim Ali Shakir. This is an open access article distributed under the Creative Commons Attribution License, which permits unrestricted use, distribution, and reproduction in any medium, provided the original work is properly cited. 
For the functions $f \in S(p, w)$ given by (1.2) and $g \in S(p, w)$ defined by

$$
g(z)=(z-w)^{p}-\sum_{n=1}^{\infty} b_{n+p}(z-w)^{n+p} \quad\left(b_{n+p} \geq 0, p \in \mathbb{N}\right),
$$

we define the Hadamard product of $f$ and $g$ by

$$
(f * g)(z)=(z-w)^{p}-\sum_{n=1}^{\infty} a_{n+p} b_{n+p}(z-w)^{n+p}
$$

For $a \in \mathbb{R}, c \in \mathbb{R} \backslash \mathbb{Z}_{0}^{-}$, with $\mathbb{Z}_{0}^{-}=\{0,-1,-2, \ldots\}, \quad 0 \leq \delta<1, \quad p \in \mathbb{N}, \quad \tau>-p$, $\alpha, \beta \in \mathbb{R}$ with $\alpha+\beta-p<1$ and $f \in S(p, w)$. The linear operator $\mathcal{L}_{\alpha, \beta, \delta}^{p, w, \tau}(a, c)$ : $S(p, w) \rightarrow S(p, w)$ (see [3]) is defined by

$$
\mathcal{L}_{\alpha, \beta, \delta}^{p, w, \tau}(a, c) f(z)=(z-w)^{p}+\sum_{n=1}^{\infty} \varphi(a, c, \alpha, \beta, \delta, \tau, n, p) a_{n+p}(z-w)^{n+p}
$$

where

$$
\varphi(a, c, \alpha, \beta, \delta, \tau, n, p)=\frac{(c)_{n}(p+1-\alpha)_{n}(p+1-\delta+\beta)_{n}(\tau+p)_{n}}{(a)_{n}(p+1)_{n}(p+1-\alpha+\beta)_{n} n !}
$$

Now, we define the class $S_{p}^{w}(\lambda, \eta, \mu, \tau, \alpha, \beta, \delta)$ consisting the functions $f \in S(p, w)$ such that

$$
\left|\frac{(z-w)\left(\mathcal{L}_{\alpha, \beta, \delta}^{p, w, \tau}(a, c) f(z)\right)^{\prime \prime \prime}-(p-2)\left(\mathcal{L}_{\alpha, \beta, \delta}^{p, w, \tau}(a, c) f(z)\right)^{\prime \prime}}{\lambda(z-w)\left(\mathcal{L}_{\alpha, \beta, \delta}^{p, w, \tau}(a, c) f(z)\right)^{\prime \prime \prime}+(\eta-\mu)\left(\mathcal{L}_{\alpha, \beta, \delta}^{p, w, \tau}(a, c) f(z)\right)^{\prime \prime}}\right|<1,
$$

where $0 \leq \lambda<1,0<\eta \leq 1,0 \leq \mu<1, p \in \mathbb{N}$ and $p>2$.

We note other studies of various other classes with different results, like, Ghanim and Darus [2], Najafzadeh and Rahimi [4], Shenan [5], Atshan and Wanas [1] and Wanas [6]. 


\section{Main Results}

In the first theorem, we find sharp coefficient estimates for the class $S_{p}^{w}(\lambda, \eta, \mu, \tau, \alpha, \beta, \delta)$.

Theorem 2.1. Let $f \in S(p, w)$. Then $f \in S_{p}^{w}(\lambda, \eta, \mu, \tau, \alpha, \beta, \delta)$ if and only if

$$
\begin{aligned}
& \sum_{n=1}^{\infty}(n+p)(n+p-1)(n+\eta-\mu+\lambda(n+p-2)) \varphi(a, c, \alpha, \beta, \delta, \tau, n, p) a_{n+p} \\
\leq & p(p-1)(\eta-\mu+\lambda(p-2)),
\end{aligned}
$$

where $0 \leq \lambda<1,0<\eta \leq 1,0 \leq \mu<1$ and $\varphi(a, c, \alpha, \beta, \delta, \tau, n, p)$ is given by (1.4).

The result is sharp for the function $f$ given by

$$
\begin{aligned}
& f(z)=(z-w)^{p} \\
& -\frac{p(p-1)(\eta-\mu+\lambda(p-2))}{(n+p)(n+p-1)(n+\eta-\mu+\lambda(n+p-2)) \varphi(a, c, \alpha, \beta, \delta, \tau, n, p)}(z-w)^{n+p} \\
& \quad(n \geq 1) . \quad
\end{aligned}
$$

Proof. Suppose that the inequality (2.1) holds true and $(z-w) \in \partial U$, where $\partial U$ denotes the boundary of $U$. Then, we find from (1.5) that

$$
\begin{aligned}
& \left|(z-w)\left(\mathcal{L}_{\alpha, \beta, \delta}^{p, w, \tau}(a, c) f(z)\right)^{\prime \prime \prime}-(p-2)\left(\mathcal{L}_{\alpha, \beta, \delta}^{p, w, \tau}(a, c) f(z)\right)^{\prime \prime}\right| \\
& -\left|\lambda(z-w)\left(\mathcal{L}_{\alpha, \beta, \delta}^{p, w, \tau}(a, c) f(z)\right)^{\prime \prime \prime}+(\eta-\mu)\left(\mathcal{L}_{\alpha, \beta, \delta}^{p, w, \tau}(a, c) f(z)\right)^{\prime \prime}\right| \\
& =\left|-\sum_{n=1}^{\infty} n(n+p)(n+p-1) \varphi(a, c, \alpha, \beta, \delta, \tau, n, p) a_{n+p}(z-w)^{n+p-2}\right| \\
& -\mid p(p-1)(\eta-\mu+\lambda(p-2))(z-w)^{p-2}-\sum_{n=1}^{\infty}(n+p)(n+p-1) \\
& \quad \times(\eta-\mu+\lambda(n+p-2)) \varphi(a, c, \alpha, \beta, \delta, \tau, n, p) a_{n+p}(z-w)^{n+p-2} \mid
\end{aligned}
$$




$$
\begin{aligned}
\leq & \sum_{n=1}^{\infty} n(n+p)(n+p-1) \varphi(a, c, \alpha, \beta, \delta, \tau, n, p) a_{n+p}(z-w)^{n+p-2} \\
& -p(p-1)(\eta-\mu+\lambda(p-2))|z-w|^{p-2} \\
& +\sum_{n=1}^{\infty}(n+p)(n+p-1) \\
& \times(\eta-\mu+\lambda(n+p-2)) \varphi(a, c, \alpha, \beta, \delta, \tau, n, p) a_{n+p}|z-w|^{n+p-2} \\
= & \sum_{n=1}^{\infty}(n+p)(n+p-1)(n+\eta-\mu+\lambda(n+p-2)) \varphi(a, c, \alpha, \beta, \delta, \tau, n, p) a_{n+p} \\
& -p(p-1)(\eta-\mu+\lambda(p-2)) \leq 0 .
\end{aligned}
$$

Hence, by maximum modulus theorem, we conclude $f \in S_{p}^{w}(\lambda, \eta, \mu, \tau, \alpha, \beta, \delta)$.

Conversely, suppose that $f \in S_{p}^{w}(\lambda, \eta, \mu, \tau, \alpha, \beta, \delta)$. Then from (1.3), we have

$$
\begin{aligned}
& \left|\frac{(z-w)\left(\mathcal{L}_{\alpha, \beta, \delta}^{p, w, \tau}(a, c) f(z)\right)^{\prime \prime \prime}-(p-2)\left(\mathcal{L}_{\alpha, \beta, \delta}^{p, w, \tau}(a, c) f(z)\right)^{\prime \prime}}{\lambda(z-w)\left(\mathcal{L}_{\alpha, \beta, \delta}^{p, w, \tau}(a, c) f(z)\right)^{\prime \prime \prime}+(\eta-\mu)\left(\mathcal{L}_{\alpha, \beta, \delta}^{p, w, \tau}(a, c) f(z)\right)^{\prime \prime}}\right| \\
& =\left|\begin{array}{l}
\sum_{n=1}^{\infty} n(n+p)(n+p-1) \varphi(a, c, \alpha, \beta, \delta, \tau, n, p) a_{n+p}(z-w)^{n+p-2} \\
p(p-1)(\eta-\mu+\lambda(p-2))(z-w)^{p-2}-\sum_{n=1}^{\infty}(n+p)(n+p-1) \\
\times(\eta-\mu+\lambda(n+p-2)) \varphi(a, c, \alpha, \beta, \delta, \tau, n, p) a_{n+p}(z-w)^{n+p-2}
\end{array}\right| \\
& <1 .
\end{aligned}
$$


So, we obtain

$$
\operatorname{Re}\left\{\begin{array}{l}
\frac{\sum_{n=1}^{\infty} n(n+p)(n+p-1) \varphi(a, c, \alpha, \beta, \delta, \tau, n, p) a_{n+p}(z-w)^{n+p-2}}{p(p-1)(\eta-\mu+\lambda(p-2))(z-w)^{p-2}-\sum_{n=1}^{\infty}(n+p)(n+p-1)} \\
\times(\eta-\mu+\lambda(n+p-2)) \varphi(a, c, \alpha, \beta, \delta, \tau, n, p) a_{n+p}(z-w)^{n+p-2}
\end{array}\right\}<1 .
$$

By letting $(z-w) \rightarrow 1^{-}$, through real values, we have

$$
\begin{aligned}
& \sum_{n=1}^{\infty}(n+p)(n+p-1)(n+\eta-\mu+\lambda(n+p-2)) \varphi(a, c, \alpha, \beta, \delta, \tau, n, p) a_{n+p} \\
\leq & p(p-1)(\eta-\mu+\lambda(p-2)) .
\end{aligned}
$$

Corollary 2.1. Let $f \in S_{p}^{w}(\lambda, \eta, \mu, \tau, \alpha, \beta, \delta)$. Then

$$
a_{n+p} \leq \frac{p(p-1)(\eta-\mu+\lambda(p-2))}{(n+p)(n+p-1)(n+\eta-\mu+\lambda(n+p-2)) \varphi(a, c, \alpha, \beta, \delta, \tau, n, p)}
$$

In the next result, we discuss extreme points for the class $S_{p}^{w}(\lambda, \eta, \mu, \tau, \alpha, \beta, \delta)$.

Theorem 2.2. Let $f_{p}(z)=(z-w)^{p}$ and

$$
\begin{aligned}
& f_{n+p}(z)=(z-w)^{p} \\
& -\frac{p(p-1)(\eta-\mu+\lambda(p-2))}{(n+p)(n+p-1)(n+\eta-\mu+\lambda(n+p-2)) \varphi(a, c, \alpha, \beta, \delta, \tau, n, p)}(z-w)^{n+p} \\
& \quad(n \geq 1) .
\end{aligned}
$$

Then $f \in S_{p}^{w}(\lambda, \eta, \mu, \tau, \alpha, \beta, \delta)$ if and only if it can be expressed in the form 


$$
f(z)=\sum_{n=0}^{\infty} \gamma_{n+p} f_{n+p}(z)
$$

where $\gamma_{n+p} \geq 0, \sum_{n=0}^{\infty} \gamma_{n+p}=1$

Proof. Let the $f$ of the form (2.3). Then

$$
\begin{aligned}
& f(z)=\gamma_{p} f_{p}(z)+\sum_{n=1}^{\infty} \gamma_{n+p}\left((z-w)^{p}\right. \\
& \left.-\frac{p(p-1)(\eta-\mu+\lambda(p-2))}{(n+p)(n+p-1)(n+\eta-\mu+\lambda(n+p-2)) \varphi(a, c, \alpha, \beta, \delta, \tau, n, p)}(z-w)^{n+p}\right) \\
& =(z-w)^{p}-\sum_{n=1}^{\infty} \frac{p(p-1)(\eta-\mu+\lambda(p-2))}{(n+p)(n+p-1)(n+\eta-\mu+\lambda(n+p-2)) \varphi(a, c, \alpha, \beta, \delta, \tau, n, p)} \\
& \quad \times \gamma_{n+p}(z-w)^{n+p} .
\end{aligned}
$$

Now,

$$
\begin{aligned}
& \sum_{n=1}^{\infty} \frac{(n+p)(n+p-1)(n+\eta-\mu+\lambda(n+p-2)) \varphi(a, c, \alpha, \beta, \delta, \tau, n, p)}{p(p-1)(\eta-\mu+\lambda(p-2))} \\
& \times \frac{p(p-1)(\eta-\mu+\lambda(p-2))}{(n+p)(n+p-1)(n+\eta-\mu+\lambda(n+p-2)) \varphi(a, c, \alpha, \beta, \delta, \tau, n, p)} \gamma_{n+p} \\
& =\sum_{n=1}^{\infty} \gamma_{n+p}=1-\gamma_{p} \leq 1 .
\end{aligned}
$$

Thus $f \in S_{p}^{w}(\lambda, \eta, \mu, \tau, \alpha, \beta, \delta)$.

Conversely, let $f \in S_{p}^{w}(\lambda, \eta, \mu, \tau, \alpha, \beta, \delta)$. It follows from Corollary 2.1 that

$$
a_{n+p} \leq \frac{p(p-1)(\eta-\mu+\lambda(p-2))}{(n+p)(n+p-1)(n+\eta-\mu+\lambda(n+p-2)) \varphi(a, c, \alpha, \beta, \delta, \tau, n, p)}(n \geq 1) .
$$


Setting

$$
\gamma_{n+p}=\frac{(n+p)(n+p-1)(n+\eta-\mu+\lambda(n+p-2)) \varphi(a, c, \alpha, \beta, \delta, \tau, n, p)}{p(p-1)(\eta-\mu+\lambda(p-2))} a_{n+p}
$$

and $\gamma_{p}=1-\sum_{n=1}^{\infty} \gamma_{n+p}$, we have

$$
f(z)=(z-w)^{p}-\sum_{n=1}^{\infty} a_{n+p}(z-w)^{n+p}
$$$$
=(z-w)^{p}-\sum_{n=1}^{\infty} \frac{p(p-1)(\eta-\mu+\lambda(p-2))}{(n+p)(n+p-1)(n+\eta-\mu+\lambda(n+p-2)) \varphi(a, c, \alpha, \beta, \delta, \tau, n, p)}
$$$$
\times \gamma_{n+p}(z-w)^{n+p}
$$

$=(z-w)^{p}-\sum_{n=1}^{\infty}\left((z-w)^{p}-f_{n+p}(z)\right) \gamma_{n+p}$

$=\left(1-\sum_{n=1}^{\infty} \gamma_{n+p}\right)(z-w)^{p}+\sum_{n=1}^{\infty} \gamma_{n+p} f_{n+p}(z)$

$=\gamma_{p} f_{p}(z)+\sum_{n=1}^{\infty} \gamma_{n+p} f_{n+p}(z)=\sum_{n=0}^{\infty} \gamma_{n+p} f_{n+p}(z)$

that is the required representation.

In the following theorem, we establish integral representation for functions belongs to the class $S_{p}^{w}(\lambda, \eta, \mu, \tau, \alpha, \beta, \delta)$.

Theorem 2.3. Let $f \in S_{p}^{w}(\lambda, \eta, \mu, \tau, \alpha, \beta, \delta)$. Then

$$
\mathcal{L}_{\alpha, \beta, \delta}^{p, w, \tau}(a, c) f(z)=\int_{0}^{z} \int_{0}^{z} \exp \left[\int_{0}^{z} \frac{(\eta-\mu) \psi\left(t_{1}\right)+p-2}{\left(t_{1}-w\right)\left(1-\lambda \psi\left(t_{1}\right)\right)} d t_{1}\right] d t_{2} d t_{3},
$$

where $|\psi(z)|<1, z \in U$. 
Proof. By putting $\frac{(z-w)\left(\mathcal{L}_{\alpha, \beta, \delta}^{p, w, \tau}(a, c) f(z)\right)^{\prime \prime \prime}}{\left(\mathcal{L}_{\alpha, \beta, \delta}^{p, w, \tau}(a, c) f(z)\right)^{\prime \prime}}=Q(z)$ in (1.5), we have

$$
\left|\frac{Q(z)-p+2}{\lambda Q(z)+\eta-\mu}\right|<1
$$

or equivalently

$$
\frac{Q(z)-p+2}{\lambda Q(z)+\eta-\mu}=\psi(z), \quad(|\psi(z)|<1, z \in U)
$$

So

$$
\frac{\left(\mathcal{L}_{\alpha, \beta, \delta}^{p, w, \tau}(a, c) f(z)\right)^{\prime \prime \prime}}{\left(\mathcal{L}_{\alpha, \beta, \delta}^{p, w, \tau}(a, c) f(z)\right)^{\prime \prime}}=\frac{(\eta-\mu) \psi(z)+p-2}{(z-w)(1-\lambda \psi(z))}
$$

after integration, we get

$$
\left.\log \left(\left(\mathcal{L}_{\alpha, \beta, \delta}^{p, w, \tau}(a, c) f(z)\right)\right)^{\prime \prime}\right)=\int_{0}^{z} \frac{(\eta-\mu) \psi\left(t_{1}\right)+p-2}{\left(t_{1}-w\right)\left(1-\lambda \psi\left(t_{1}\right)\right)} d t_{1} .
$$

Therefore

$$
\left(\mathcal{L}_{\alpha, \beta, \delta}^{p, w, \tau}(a, c) f(z)\right)^{\prime \prime}=\exp \left[\int_{0}^{z} \frac{(\eta-\mu) \psi\left(t_{1}\right)+p-2}{\left(t_{1}-w\right)\left(1-\lambda \psi\left(t_{1}\right)\right)} d t_{1}\right]
$$

By integration once again, we have

$$
\left(\mathcal{L}_{\alpha, \beta, \delta}^{p, w, \tau}(a, c) f(z)\right)^{\prime}=\int_{0}^{z} \exp \left[\int_{0}^{z} \frac{(\eta-\mu) \psi\left(t_{1}\right)+p-2}{\left(t_{1}-w\right)\left(1-\lambda \psi\left(t_{1}\right)\right)} d t_{1}\right] d t_{2} .
$$

Also, after integration, we conclude that

$$
\mathcal{L}_{\alpha, \beta, \delta}^{p, w, \tau}(a, c) f(z)=\int_{0}^{z} \int_{0}^{z} \exp \left[\int_{0}^{z} \frac{(\eta-\mu) \psi\left(t_{1}\right)+p-2}{\left(t_{1}-w\right)\left(1-\lambda \psi\left(t_{1}\right)\right)} d t_{1}\right] d t_{2} d t_{3}
$$

and this the required result.

Theorem 2.4. If $f \in S_{p}^{w}(\lambda, \eta, \mu, \tau, \alpha, \beta, \delta)$, then $f$ is starlike of order $\theta$ 
$(0 \leq \theta<p)$ in the disk $|z-w|<r_{1}$, where

$$
r_{1}=\inf _{n}\left\{\frac{(n+p)(p-\theta)(n+p-1)(n+\eta-\mu+\lambda(n+p-2)) \varphi(a, c, \alpha, \beta, \delta, \tau, n, p)}{p(p-1)(n+p-\theta)(\eta-\mu+\lambda(p-2))}\right\}^{\frac{1}{n}} .
$$

The result is sharp for the function $f$ given by (2.2).

Proof. It is sufficient to show that

$$
\left|\frac{(z-w) f^{\prime}(z)}{f(z)}-p\right| \leq p-\theta \text { for }|z-w|<r_{1} .
$$

But

$$
\left|\frac{(z-w) f^{\prime}(z)}{f(z)}-p\right|=\left|\frac{-\sum_{n=1}^{\infty} n a_{n+p}(z-w)^{n+p}}{(z-w)^{p}-\sum_{n=1}^{\infty} a_{n+p}(z-w)^{n+p}}\right| \leq \frac{\sum_{n=1}^{\infty} n a_{n+p}|z-w|^{n}}{1-\sum_{n=1}^{\infty} a_{n+p}|z-w|^{n}} .
$$

Thus (2.4) will be satisfied if

$$
\frac{\sum_{n=1}^{\infty} n a_{n+p}|z-w|^{n}}{1-\sum_{n=1}^{\infty} a_{n+p}|z-w|^{n}} \leq p-\theta,
$$

or if

$$
\sum_{n=1}^{\infty} \frac{(n+p-\theta)}{(p-\theta)} a_{n+p}|z-w|^{n} \leq 1
$$

with the aid of (2.1), (2.5) is true if

$$
\begin{aligned}
& \frac{(n+p-\theta)}{(p-\theta)}|z-w|^{n} \\
\leq & \frac{(n+p)(n+p-1)(n+\eta-\mu+\lambda(n+p-2)) \varphi(a, c, \alpha, \beta, \delta, \tau, n, p)}{p(p-1)(\eta-\mu+\lambda(p-2))},
\end{aligned}
$$


or equivalently

$$
\begin{aligned}
&|z-w| \\
& \leq\left\{\frac{(n+p)(p-\theta)(n+p-1)(n+\eta-\mu+\lambda(n+p-2)) \varphi(a, c, \alpha, \beta, \delta, \tau, n, p)}{p(p-1)(n+p-\theta)(\eta-\mu+\lambda(p-2))}\right\}^{\frac{1}{n}} \\
& \quad(n \geq 1),
\end{aligned}
$$

which follows the result.

Theorem 2.5. If $f \in S_{p}^{w}(\lambda, \eta, \mu, \tau, \alpha, \beta, \delta)$, then $f$ is convex of order $\theta$ $(0 \leq \theta<p)$ in the disk $|z-w|<r_{2}$, where

$$
r_{2}=\inf _{n}\left\{\frac{(p-\theta)(n+p-1)(n+\eta-\mu+\lambda(n+p-2)) \varphi(a, c, \alpha, \beta, \delta, \tau, n, p)}{(p-1)(n+p-\theta)(\eta-\mu+\lambda(p-2))}\right\}^{\frac{1}{n}} .
$$

The result is sharp for the function $f$ given by (2.2).

Proof. It is sufficient to show that

$$
\left|\frac{(z-w) f^{\prime \prime}(z)}{f^{\prime}(z)}+1-p\right| \leq p-\theta \text { for }|z-w|<r_{2} .
$$

But

$$
\begin{aligned}
\left|\frac{(z-w) f^{\prime \prime}(z)}{f^{\prime}(z)}+1-p\right| & =\left|\frac{-\sum_{n=1}^{\infty} n(n+p) a_{n+p}(z-w)^{n+p-1}}{p(z-w)^{p-1}-\sum_{n=1}^{\infty}(n+p) a_{n+p}(z-w)^{n+p-1}}\right| \\
& \leq \frac{\sum_{n=1}^{\infty} n(n+p) a_{n+p}|z-w|^{n}}{p-\sum_{n=1}^{\infty}(n+p) a_{n+p}|z-w|^{n}} .
\end{aligned}
$$


Thus (2.6) will be satisfied if

$$
\frac{\sum_{n=1}^{\infty} n(n+p) a_{n+p}|z-w|^{n}}{p-\sum_{n=1}^{\infty}(n+p) a_{n+p}|z-w|^{n}} \leq p-\theta,
$$

or if

$$
\sum_{n=1}^{\infty} \frac{(n+p)(n+p-\theta)}{p(p-\theta)} a_{n+p}|z-w|^{n} \leq 1,
$$

with the aid of (2.1), (2.7) is true if

$$
\begin{aligned}
& \frac{(n+p)(n+p-\theta)}{p(p-\theta)}|z-w|^{n} \\
\leq & \frac{(n+p)(n+p-1)(n+\eta-\mu+\lambda(n+p-2)) \varphi(a, c, \alpha, \beta, \delta, \tau, n, p)}{p(p-1)(\eta-\mu+\lambda(p-2))},
\end{aligned}
$$

or equivalently

$$
\begin{array}{r}
|z-w| \leq\left\{\frac{(p-\theta)(n+p-1)(n+\eta-\mu+\lambda(n+p-2)) \varphi(a, c, \alpha, \beta, \delta, \tau, n, p)}{(p-1)(n+p-\theta)(\eta-\mu+\lambda(p-2))}\right\}^{\frac{1}{n}} \\
\quad(n \geq 1),
\end{array}
$$

which follows the result.

\section{References}

[1] W. G. Atshan and A. K. Wanas, Subclass of $p$-valent analytic functions with negative coefficients, Adv. Appl. Math. Sci. 11(5) (2012), 239-254.

[2] F. Ghanim and M. Darus, On new subclass of analytic univalent function with negative coefficient, I, Int. J. Contemp. Math. Sci. 3(27) (2008), 1317-1329.

[3] R. Kargar, A. Bilavi, S. Abdolahi and S. Maroufi, A class of multivalent analytic functions defined by a new linear operator, J. Math. Comp. Sci. 8 (2014), 326-334. https://doi.org/10.22436/jmcs.08.04.01 
[4] Sh. Najafzadeh and A. Rahimi, Application of differential subordination on $p$-valent functions with a fixed point, Gen. Math. 17(4) (2009), 149-156.

[5] J. M. Shenan, On a subclass of p-valent prestarlike functions with negative coefficient defined by Dziok-Srivastava linear operator, Int. J. Open Probl. Complex Anal. 3(3) (2011), 24-35.

[6] A. K. Wanas, Some properties of a certain class of multivalent analytic functions with a fixed point, International Journal of Innovative Science, Engineering \& Technology 1(9) (2014), 336-339. 